\title{
Preservative Realism and Its Discontents: Revisiting Caloric
}

\author{
Hasok Chang ${ }^{\dagger}$
}

\begin{abstract}
A popular and plausible response against Laudan's "pessimistic induction" has been what I call "preservative realism," which argues that there have actually been enough elements of scientific knowledge preserved through major theory-change processes, and that those elements can be accepted realistically. This paper argues against preservative realism, in particular through a critical review of Psillos's argument concerning the case of the caloric theory of heat. Contrary to his argument, the historical record of the caloric theory reveals that beliefs about the properties of material caloric, rejected by subsequent theories, were indeed central to the successes of the caloric theory. Therefore caloric remains a favorable case for Laudan. Further, I argue that even confirmed cases of preservation do not warrant an inference to truth.
\end{abstract}

1. Introduction. As scientific realists attempt to counter Larry Laudan's "pessimistic induction from the history of science" (1981), ${ }^{1}$ perhaps the most promising line of attack is what I will call "preservative realism" in this paper. According to the preservative realists, Laudan exaggerates the discontinuities shown in the historical record of science; contrary to Laudan's arguments, there are significant elements of successful scientific theories that get preserved even through revolutionary upheavals, and those preserved elements are what we can accept in a realist way. There are several varieties of preservative realism, including John Worrall's structural realism (1989) and Philip Kitcher's idea that "working posits" are immune to the pessimistic induction (1993, esp. 149).

$\dagger$ To contact the author, please write to: Department of Science and Technology Studies, University College London, Gower Street, London WC1E 6BT, United Kingdom; e-mail: h.chang@ucl.ac.uk.

1. Although the basic idea of the pessimistic induction was not original to Laudan (it was at least implicit in Thomas Kuhn's discussion of incommensurability, if not also in other, earlier works), it was Laudan's sharp formulation of the idea that brought the point forcefully into the realism literature.

Philosophy of Science, 70 (December 2003) pp. 902-912. 0031-8248/2003/7005-0004\$10.00

Copyright 2003 by the Philosophy of Science Association. All rights reserved. 
I wish to argue that preservative realism does not work. Since that is a task that would require much more than a brief paper, here I will concentrate on the critique of one particular preservative-realist argument, which will also reveal some symptomatic problems with the general strategy.

2. Psillos on the Caloric Theory of Heat. The particular argument I want to examine in this paper is the discussion of the caloric theory by Stathis Psillos (1999, 115-130; also Psillos 1994). There are obvious reasons for choosing Psillos's work as the focus of my discussion. Not only is he a major commentator on the realism debate, but he is also one of the very few scientific realists who have engaged with the pessimistic induction in a serious historical vein. In his admirable recent book on realism, he gives a detailed examination of two of Laudan's prime cases, optics and the caloric theory. I will discuss the latter because that happens to be a historical case that I know well, and also because I think the former has received a bit more historical attention so far.

Psillos's conclusion for the caloric case is as follows:

$[\mathrm{N}]$ ot all cases of abandoned terms are troublesome. The serious cases concern terms which were indeed central in some genuinely successful theory. . . . When some abandoned term had not been central, realists should not be required to show how it can possibly be referential. "Caloric," simply, was not such a central term. (Psillos 1999, 129130)

More generally, Psillos wants to maintain a disjunction between "the theoretical constituents of past genuine successful theories that made essential contributions to their successes," and other constituents that were included in the successful theories but did not actually do useful work $(1999,110)$. That disjunction is indeed necessary as a basis of his main strategy against the pessimistic induction, which he calls the "divide et impera move":

$[\mathrm{W}]$ hen a theory is abandoned, its theoretical constituents ... should not be rejected en bloc.... [I]f it turns out that the theoretical constituents that were responsible for the empirical success of otherwise abandoned theories are those that have been retained in our current scientific image, then a substantive version of scientific realism can still be defended. (Psillos 1999, 108)

Psillos argues that the "laws of the caloric theory can be deemed to be approximately true independently of the referential failure of 'caloric"' (1999, 127).

Let us see if the history of the caloric theory supports the preservative strategy, at first taking the history as Psillos tells it. He lists the following as 
the "three stages in the development of the caloric theory": laws of calorimetry, the adiabatic law, and Carnot's theory of heat engines (Psillos 1994, 168-179, 183; Psillos 1999, 125). Setting aside the point that anyone with a detailed appreciation of the scientific work in the heyday of the caloric theory will be puzzled by this selection, I am not even certain that they actually help Psillos's case. Let us examine the three items one by one.

Calorimetry was developed long before the caloric theory, mostly by experimental considerations. As Psillos himself notes (1994, 168), it is a practice that can be, and was, supported by the most phenomenological of theories. All that it requires theoretically are: the assumption of heat conservation in a particular class of phenomena, namely, the simple mixing of two substances in which no chemical (or other strange) reactions take place; and a concept of specific heat, phenomenologically understood as the amount of heat required in effecting a unit increment in temperature. Nothing in calorimetry actually depends on the caloric theory; if that is Psillos's point, then the point is correct. But if calorimetry was not a part of the caloric theory, the preservation of calorimetric practices into later science is neither here nor there when we are trying to see whether the case of caloric bears out Laudan's contention.

The second stage in Psillos's picture of the development of caloric theory is equally problematic, but in a very different way. The derivation of the law of adiabatic expansion of gases did occur squarely within the caloric tradition. More specifically, it was the work of Pierre-Simon Laplace and his school, who took caloric as a "discrete fluid" made up of point-like particles that exerted repulsive forces on each other, and attractive forces on particles of ordinary matter (see Fox 1971, especially ch. 5). But this seems to me rather a perfect case for Laudan, because the Laplacian derivations did rely on particular ontological assumptions about the nature of caloric, as I will discuss further in the next section. The fact that the Laplacians got the "correct" (still preserved) formula on the basis of the now-rejected ontology should alarm the realists, not reassure them.

Psillos argues that in the famous case of calculating the correction to Newton's theoretical value for the speed of sound, "Laplace's account does not explicitly rest on any particular representation of heat, although he happened to be an advocate of the caloric theory." That is a debatable point regarding Laplace's calculations, but the point is not debatable when it comes to the explanatory rationale behind Laplace's calculations. As Psillos himself states: "He assumed that there was some quantity of latent heat which was released from the compression of the air" (1999, 120). At that time, the only plausible explanation of adiabatic heating was to understand it as the disengagement of caloric from ordinary matter, caused by mechanical compression. As the standard kinetic theory at this time understood heat as vibrations of molecules around fixed points 
even in gases, it could not give a plausible explanation of adiabatic heating.

Psillos's third stage, Sadi Carnot's theory of heat engines, is also problematic, in a complex way. First of all, Carnot's work was not central to the caloric tradition. It was created in the twilight of the caloric theory, and most of the leading caloric theorists paid little attention to it. Perhaps even more importantly, the character of Carnot's theory was also very anomalous within the caloric tradition. Like the classical thermodynamic theory that developed later and built on his work, Carnot's theory was already macroscopic-phenomenalistic to the point of abandoning all of the Laplacian microphysics of particles and forces. Even where Carnot used the adiabatic law, he merely used it as an empirical regularity without relying on the Laplacian theory behind its derivation. Carnot's lasting achievement was his novel method of tracing the macroscopic workings of the ideal heat engine, not anything based on his hesitant acceptance of the caloric theory. Psillos himself makes a similar point, but what it really shows is that the success of Carnot's theory is just as irrelevant as calorimetry to the issues at hand.

To the extent that Carnot was a calorist, his work was a failure. In the places where Carnot did make use of the central assumptions of the caloric theory, his theory was not preserved but significantly revised. That can be seen with the utmost clarity in the works of William Thomson (Lord Kelvin), who first elaborated thermodynamics in the late 1840s, in Carnot's vein, and then had to modify the theory significantly (into the form we are now familiar with) when he adopted energy conservation under James Joule's influence. The essence of the later thermodynamic understanding of the heat engine was that heat was not conserved, but destroyed and converted into mechanical work. That later picture was also incompatible with Carnot's idea that the mechanical work was generated by the movement of caloric from a hotter to a colder place, in a process analogous to the generation of power by the downward flow of water in a water-wheel. All this amounted to the negation of the main thing that Carnot believed about the caloric theory. The preserved elements of Carnot's theory, such as the anticipation of the second law of thermodynamics, were just those parts that were unrelated to the tenets of the caloric theory.

To summarize, the historical material highlighted by Psillos ends up falling into the following categories, neither of which is effective in supporting his case.

(1) Beliefs and practices that were not really part of the caloric theory: whether these were preserved or not is irrelevant to the issue at hand. 
(2) Theoretical beliefs within the caloric theory that were later rejected: to the extent that these had led to successful empirical results, they are just grist for Laudan's mill.

3. The Real Successes of the Caloric Theory. Leaving behind the history of the caloric theory as Psillos presents it, I would now like to evaluate the cogency of the preservative-realist claim with respect to the real history of the caloric theory. But before going into the history, the philosophical thesis itself needs to be refined. Psillos argues that the empirical success of the caloric theory was not due to the belief in the material reality of heat. I do not think that the empirical success of any theory could credibly be attributed to a belief at that level of vagueness. Where things get more interesting is at the next level up in specificity: what kind of material substance is caloric; what are its properties, and what is the nature of its interactions with other substances? If we may avoid an obsession with reference, Laudan's point can be reformulated as follows: the caloric theory was empirically successful, but the assumptions about the nature of caloric that were responsible for that success have been rejected as incorrect. I think that claim is born out amply by the history.

In looking at the history, we must start by compiling a list of the successes of the caloric theory. We cannot take Psillos's version of the history, because there is little resemblance between what he describes as "the laws of the caloric theory" and the most important aspects of the actual caloric theories that once dominated the scientific landscape of Western Europe. ${ }^{2}$ At least in the case of caloric, Psillos does not entirely escape the syndrome lamented by Paul Feyerabend $(1975,19)$ as follows: "A little brainwashing will go a long way in making the history of science duller, simpler, more uniform, more 'objective' and more easily accessible to treatment by strict and unchangeable rules." Stephen Brush would give a diagnosis of "precursoritis": in surveying the history of the caloric theory, Psillos has only picked out the items that have a clear appearance of being precursors of our modern beliefs. Even if this exercise were successful, it could only serve up a vacuous proof that those elements of past science that have survived into modern science have, indeed, survived.

I am happy to follow the definition of "success" that Psillos seems to be working with: a theory is successful when it explains phenomena (however we construe "explanation"), and produces better explanations than avail-

2. In order to make this point fully, I would need to bring the voluminous historical literature on caloric to bear on the debate, which I cannot do here. The most convenient brief exposition of the history of caloric is Lilley 1948, which Psillos refers to. The most reliable and extensive discussion can be found in Fox 1971. 
able competing theories can. But applying that criterion of success to the history of caloric theory yields a very different list of successes from that given by Psillos. The highlights of success in the actual history include the explanations of the following: the flow of heat toward equilibrium, the expansion of matter by heating, latent heat in changes of state, the elasticity of gases and the fluidity of liquids, the heat released and absorbed in chemical reactions, combustion, the radiation of heat, and the gas laws. In order to distinguish between the "idle" and "essentially contributing" constituents of the caloric theory, we need to discern which elements of the theory were responsible for those successful explanations. After that, we can see to what extent the essentially contributing constituents were preserved in later theories.

The initial attraction of the material theory of heat was in the easy qualitative explanations it gave of the most basic thermal phenomena. The most crucial theoretical assumption was that heat was a "self-repulsive" (or "elastic," or "expansive") substance, while it was attracted to ordinary matter. When combined with the idea that temperature was the density of caloric, this basic system of forces yielded nice explanations of why heat tended to flow from warmer to colder places, and why most things expanded when they were heated. In the late-eighteenth century these basic facts were not easily explained without recourse to the caloric theory; attempts based on caloric's main competitor, namely, the primitive idea that heat was a form of motion, were generally considered to be inferior to the caloric-based explanations.

After that start, the first major theoretical elaboration of the caloric theory was the postulation that caloric existed in two different states: sensible and latent. ${ }^{3}$ In its latent state, caloric was conceived to be unable to affect the thermometer or our senses. As Psillos notes, the origin of this version of caloric theory was in chemistry, particularly the works of Joseph Black and Antoine-Laurent Lavoisier. Black's most visible achievement in heat theory was the explanation that changes of state (e.g., melting, freezing, boiling and condensation) were caused by the addition or withdrawal of latent heat. This idea explained not only why the addition or subtraction of heat did not result in temperature changes during changes of state, but also explained the fundamental changes of physical properties (e.g., fluidity and elasticity) as a consequence of the chemical combination (or dissociation) of matter with large amounts of caloric. The assumption of the two states of caloric was absolutely central to Black's explanationsnot at all an idle piece of metaphysics.

3. Here I am neglecting the important tradition of William Irvine's caloric theory, according to which there was only one state of caloric. For a discussion of the Irvinist theory, see Fox 1971, 25-28, and passim. 
The two-state assumption was firmly and explicitly based on the materialistic conception of caloric in Lavoisier's great work, which brings us to the maturity of the caloric theory. Lavoisier unequivocally identified caloric as a chemical element, listing it prominently (just above oxygen) in the table of chemical elements in his definitive textbook of the new chemistry, Traité élémentaire de chimie, published in 1789. ${ }^{4}$ Lavoisier's conception of caloric as a chemical substance provided an attractive and straightforward framework for the explanation of the heat absorbed and released in various chemical reactions, which the dynamic theory of heat at that time could not even come close to explaining. For Lavoisier, latent heat was caloric when chemically combined with matter; if that chemical bond was broken, free caloric was generated, manifesting itself as sensible heat. The most celebrated instance of this, of course, was the production of heat in combustion: the oxygen "base" combines with combustible substances, leaving behind the prodigious amount of caloric with which it had been combined. Lavoisier's conception of oxygen gas as a chemical compound made up of oxygen base and a great deal of caloric was firmly rooted in the general calorist conception of the three states of matter, according to which it was the addition of latent caloric that converted solids into liquids, and liquids into gases. Therefore his explanation of combustion constituted a particularly satisfying synthesis.

The explanatory centrality of caloric conceived as a particular type of substance becomes even clearer when we consider a couple of further major stages in the history of the caloric theory. Of immediate relevance here, though sometimes neglected even by historians, is radiant heat, which was studied with experimental precision since about 1790. In short, caloric presented itself as the most obvious conceptual means of understanding the radiation of heat: radiant heat was merely caloric being thrown about between objects at extremely high speeds. People who did not believe in the existence of caloric were left seriously confounded by radiant-heat phenomena. Count Rumford, undoubtedly the most formidable opponent of the caloric theory at the time, was forced to adopt a highly idiosyncratic theory of ether vibrations in order to make sense of radiant heat. Rumford's theory was in fact quite cogent in my opinion, but it was almost universally rejected or neglected because of its unusual features such as the doctrine that the radiation of cold was equally real as the radiation of heat (see Chang 2002). In the best scientific consensus of the day, the caloric theory explained radiant heat, and did it much better than any easily conceivable competitors.

Finally, we must consider the clear height of theoretical sophistication in the caloric tradition, namely Laplace's work in the 1820s. Psillos does

4. For a detailed discussion of Lavoisier's caloric theory, see Morris 1972. 
discuss Laplace's work, but curiously only focuses on his success regarding the speed of sound. ${ }^{5}$ Laplace's caloric theory explained a good deal of important phenomena (the speed of sound, the adiabatic gas law, and the regularities that are summarized by the ideal gas law), and it explained these things better than any other competing theory did. Until the advent of the molecular-kinetic theory of gases in the second half of the nineteenth century, there were no viable contenders to the Laplacian theory of gases.

What was responsible for Laplace's success? Once again, contrary to what Psillos implies, assumptions about the material nature of caloric played a crucial role. Laplace's central premises were that the caloric fluid was made up of point-like particles of caloric, and that the caloric particles, most of which were contained within molecules of matter, repelled each other with a force that was a function of distance only. Temperature was defined as the density of "free caloric of space," which was a small amount of caloric flying around in intermolecular spaces, dislodged from the molecules by intercaloric repulsion. Since Laplace did not know the precise form of the intercaloric force function, he also needed other assumptions such as the following: the force is negligible at any sensible distances; each molecule in a gas in equilibrium contains the same amount of caloric; in equilibrium, the caloric-filled molecules are spherical and stationary; and so on (see Chang 2001, 265-267). Could all these premises for Laplace's derivation of the gas laws be understood in any sense as approximately true according to modern theories? If so, I would be very surprised. The Laplacian metaphysics of mutually repelling caloric particles has been completely and unhesitatingly rejected by modern science; the gas laws have now been derived from entirely different assumptions.

I have now given a very quick survey of some of the most significant and undisputed successes of the most orthodox line of the caloric theory. Almost all of them are neglected by Psillos; each of them tends to support the pessimistic induction, and fits Psillos's story only with great difficulty. The various assumptions about the nature of caloric and its interaction with ordinary matter did perform essential work in producing successful explanations, and they were clearly rejected by later science. (In addition, it could easily be argued that the assumption of the materiality of caloric was central to these explanatory successes, because it is difficult to see how the various subsidiary assumptions about the nature of that substance can be translated into sensible terms that do not involve the assumption of its materiality.)

5. Psillos explicitly states that he will not treat Laplace's work on caloric in the 1820 s, and states that this neglect will not affect the argument of the chapter $(1999,306-307$ n. 3). I think it does. 
One last point needs to be made before closing this section. Psillos $(1999,116-119)$ maintains that "the scientists of this period were not committed to the truth of the hypothesis that the cause of heat was a material substance," although they generally preferred the caloric theory over the kinetic theory and made use of the caloric theory in giving explanations. $\mathrm{He}$ cites various statements from Black and Lavoisier expressing reservations about accepting the truth of the material theory as a certainty. Although the statements he quotes are genuine, they need to be understood in context. First of all, Lavoisier and especially Black expressed that sort of caution about all theories, not just the caloric theory. It is also difficult to take the expressed caution too seriously, especially in Lavoisier's case; as Robert Morris $(1972,31)$ notes, from a man who put caloric into his table of chemical elements, the theoretical caution comes across as mere lip service to epistemic prudence, which was fashionable during that period. In any case, what matters for our present purposes is whether the materiality of caloric was in fact essential for the explanations of phenomena that were accepted, not how willing the scientists were to claim to know anything for certain.

4. The Long Road From Preservation to Realism. So far, I have argued that the real history of the caloric theory does support Laudan's pessimistic induction, despite Psillos's best efforts to show the contrary. One case, of course, does not have much force as empirical evidence. Much more careful historical work will be needed if we are to have a good sense of how much of the history of science supports Laudan's contention, and no such general conclusions can be drawn from my discussion of the caloric theory. However, in this final section of the paper I would like to add some general reflections regarding the plausibility of what I termed "preservative realism" in the introduction. The following arguments are not put forward as points proven by the case of caloric, but as general points that should be obvious without any reliance on detailed historical evidence, though they were stimulated by the consideration of the case of caloric. In this section I also depart from a critique of Psillos, who has further positive arguments for realism that need to be considered in their own right. ${ }^{6}$

Generally speaking, as long as there is something that is typically preserved from successful theories that are later discredited, the preservationist strategy has a fighting chance. Therefore it becomes important to consider what kinds of things do tend to get preserved through major theoretical changes, and to see whether the preservation of those things can support realism.

6. I thank Professor Ernan McMullin for stressing this last point. 
The survival of a good amount of old observational data would seem to be a fairly typical feature of scientific change, despite the highly publicized extreme cases in which the data themselves did change. The next category that is a good candidate for preservation is phenomenological laws, which represent the data into convenient mathematical relationships without specifying causes, mechanisms, and far-reaching theoretical principles; here we come to things like the laws of calorimetry, compatible with any theory of heat that allows conservation in simple mixtures. But neither of those categories will give the realists what they want, and the majority of antirealists, including constructive empiricists, will not be disturbed by the preservation of known observable phenomena or mere regularities found among them.

What else is typically preserved through theoretical change? There are two further categories and these are also not helpful to the realist, since they are both determined by our own habits of thinking, not by anything to do with mind-independent nature. First, there are techniques of representation and reasoning, including certain mathematical methods. Then there are deep-seated metaphysical commitments, such as the desire for a conservation principle. ${ }^{7}$ But these metaphysical propositions are things that "can be held true come what may," to borrow Quine's phrase that was meant to apply more broadly. The fact that certain representational techniques and metaphysical beliefs have been maintained by most European scientists for the past few centuries is, in itself, no indication of the way the world is.

That, as far as I can see, is the extent of the types of elements of scientific knowledge that are preserved with good enough frequency. Structural realists would object, and argue that they have identified something that survives over and above facts and habits. But so far, I do not think that they have produced many examples of lasting "structures," other than the structures of certain data-sets that are expressed in phenomenological laws, and perhaps the very abstract mathematical structures that modern physicists have a habit of applying to whatever they can get their hands on.

The foregoing consideration points to the most fundamental problem with preservative realism: even when we do have preservation, what we are allowed to infer from it is not clear at all. The uncertainty arises from the fact that there are several different reasons for which elements of scientific knowledge may be preserved. Beliefs or practices may be preserved either because nature continually speaks in favor of them, or because our own

7. Over the centuries scientists have believed in various conservation principles. Most of them were empirically refuted and rejected, but that has not stopped scientists from simply moving on to some other conservation principle. Thus we had the conservation of mass, of motion (Descartes), of vis viva (Leibniz), of heat, then of energy. 
cognitive limitations confine us to them, or because we just want to keep them. The inference from preservation to truth can be valid only if the latter two possibilities can be ruled out. Even extraordinary cases of preservation, in themselves, do not necessarily show anything beyond human limitations, or conservatism assisted by enough obstinacy and ingenuity. Preservation is far from a sufficient condition for realist acceptance.

I conclude with a metaphor designed to express this last point. The metaphor sees the development of scientific knowledge as a process of putting up a building. A building will collapse if its design goes against the laws of nature in certain crucial ways; however, if we always work certain features into the buildings we build (such as external decoration, or even certain structural aspects), that does not necessarily mean that those constant features are linked to laws of nature in any straightforward way. Likewise, in building scientific knowledge the systems we construct will collapse if they disagree with nature in certain crucial ways; however, certain features being constantly present does not mean that we can read off anything inherent about nature from them. Even if our cognitive activities are stable, we may not be able to fathom the reasons for that stability.

\section{REFERENCES}

Chang, Hasok (2001), "Spirit, Air, and Quicksilver: The Search for the 'Real' Scale of Temperature", Historical Studies in the Physical and Biological Sciences 31: 249284.

(2002), "Rumford and the Reflection of Radiant Cold: Historical Reflections and Metaphysical Reflexes", Physics in Perspective 4: 127-169.

Feyerabend, Paul K. (1975), Against Method. London: New Left Books.

Fox, Robert (1971), The Caloric Theory of Gases from Lavoisier to Regnault. Oxford: Oxford University Press.

Kitcher, Philip (1993), The Advancement of Science. Oxford: Oxford University Press.

Laudan, Larry (1981), "A Confutation of Convergent Realism", Philosophy of Science 48: $19-49$.

Lilley, S. (1948), "Attitudes to the Nature of Heat about the Beginning of the Nineteenth Century", Archives Internationales d'Histoire des Sciences 1: 630-639.

Morris, Robert J. (1972), "Lavoisier and the Caloric Theory", British Journal for the History of Science 6: 1-38.

Psillos, Stathis (1994), "A Philosophical Study of the Transition from the Caloric Theory of Heat to Thermodynamics: Resisting the Pessimistic Meta-Induction", Studies in the History and Philosophy of Science 25: 159-190. (1999), Scientific Realism: How Science Tracks Truth. London and New York: Routledge.

Worrall, John (1989), "Structural Realism: The Best of Both Worlds?”, Dialectica 43: 99124. 